\title{
Minkowski Symmetrizations of Star Shaped Sets
}

\author{
D.I. Florentin, A. Segal
}

\begin{abstract}
We provide sharp upper bounds for the number of symmetrizations required to transform a star shaped set in $\mathbb{R}^{n}$ arbitrarily close (in the Hausdorff metric) to the Euclidean ball.
\end{abstract}

\section{Introduction and results}

A non empty compact set $K \subset \mathbb{R}^{n}$ is called star shaped if $x \in K$ implies $[0, x] \subseteq K$. We denote the family of star shaped sets in $\mathbb{R}^{n}$ by $T^{n}$. Recall that given a set $K$ and a direction $u \in S^{n-1}$, it's Minkowski symmetral is defined to be

$$
M_{u}(K)=\frac{K+R_{u} K}{2}
$$

where $R_{u}$ is the reflection with respect to the hyperplane $u^{\perp}$.

The Minkowski symmetrization $M_{u}$ results in a set that is symmetric with respect to the hyperplane $u^{\perp}$, thus it is natural to expect that successive applications of this procedure in different directions yield a sequence of sets that convergences in some sense to the Euclidean ball. This is indeed known in the case where $K$ is convex. Moreover, there are estimates regarding the convergence rate. Bourgain, Lindenstrauss and Milman [1] obtained the first quantitative estimate for the convergence rate of Minkowski symmetrizations. They found a function $n_{0}:(0,1) \rightarrow \mathbb{N}$ satisfying:

Theorem 1.1 (Bourgain, Lindenstrauss, Milman). Let $\varepsilon \in(0,1)$ and let $n \in \mathbb{N}$ such that $n_{0}(\varepsilon) \leq n$. If $K \in \mathcal{K}^{n}$ is a convex body with mean width $M^{*}(K)=M_{0}$, then there exist cn $(C(\varepsilon)+\log n)$ Minkowski symmetrizations transforming $K$ into a (convex) body $\tilde{K}$, such that

$$
(1-\varepsilon) M_{0} D_{n} \subset \tilde{K} \subset(1+\varepsilon) M_{0} D_{n}
$$

Here $c$ is some positive constant, and the functions $C(\varepsilon), n_{0}(\varepsilon)$ are of the order $\left(\frac{1}{\varepsilon}\right)^{\frac{c}{\varepsilon^{2}}}$. 
In [2] Klartag improved Theorem 1.1, and also removed the restriction $n_{0} \leq n$, thus providing the first truely isometric result in all dimensions:

Theorem 1.2 (Klartag). Let $n \geq 2$ and $\varepsilon \in(0,1 / 2)$. If $K \in \mathcal{K}^{n}$ is a convex set with mean width $M^{*}(K)=M_{0}$, then there exist $c n|\log (\varepsilon)|$ Minkowski symmetrizations transforming $K$ into $\tilde{K}$, such that

$$
(1-\varepsilon) M_{0} D_{n} \subseteq \tilde{K} \subseteq(1+\varepsilon) M_{0} D_{n},
$$

where $c$ is some universal constant.

In this note we extend Klartag's theorem to $T^{n}$. Namely, we show the following:

Theorem 1.3. Let $n \geq 2$ and $\varepsilon \in(0,1 / 2)$. If $K \in T^{n}$ is a star shaped set with mean width $M^{*}(K)=M_{0}$, then there exist $C n|\log (\varepsilon)|$ Minkowski symmetrizations transforming $K$ into $\tilde{K}$, such that

$$
(1-\varepsilon) M_{0} D_{n} \subseteq \tilde{K} \subseteq(1+\varepsilon) M_{0} D_{n},
$$

where $C$ is some universal constant.

The proof of Theorem 1.3 consists of three steps. First, we make sure that the body at hand contains a small ball around the origin (Lemma 2.1). Next, we consider Minkowski symmetrizations of the convex hull of our star shaped body. As mentioned above, for convex bodies Klartag showed how many steps are required in order to bring a body isometrically close to the Euclidean ball. We apply these symmetrizations, and get a body whose convex hull lies between two balls of very similar radii. This can only happen if the body contains some " $\varepsilon$-net" of the inner ball's sphere (Lemma 2.2 . In the third and final step, we use this fact to increase the radius of the small ball, which was obtained in the first step.

Notations: The support function of a (not necessarily convex) body $K$ is defined by $h_{K}(u)=\sup \{\langle x, u\rangle \mid x \in K\}$. The width, or mean width, of a star shaped set $K$ is defined to be $M^{*}(K)=\int_{S^{n-1}} h_{K} d \sigma$, where $\sigma$ is the normalized Haar measure on the sphere.

\section{Proof of the Theorem}

Our first step is to generate (using Minkowski symmetrizations) a small ball inside a (non trivial) star shaped set.

Lemma 2.1. Let $n \geq 2, K_{0} \in T^{n}$, and let $M_{0}=M^{*}\left(K_{0}\right)>0$. Then there exist $c_{1} n$ Minkowski symmetrizations transforming $K_{0}$ into $K_{1}$, such that

$$
\frac{c_{2}}{\sqrt{n}} D_{n} \subseteq \frac{K_{1}}{M_{0}},
$$


where $c_{1}, c_{2}$ are some universal constants (in fact $c_{1}=c$ of Theorem 1.2).

Proof. Let $R \geq M_{0}>0$ be the minimal radius of a centered ball enclosing $K_{0}$. Then there exists some $u \in S^{n-1}$ such that $I_{0}=[0, R u] \subseteq K_{0}$. Let $\varepsilon=1 / e$. By Theorem 1.2 applied to $I_{0}$, there exist $N_{1}=c n$ Minkowski symmetrizations $M_{u_{1}} \ldots M_{u_{N_{1}}}$ which transform the interval $I_{0}$ into a convex body $I_{1}$ satisfying

$$
(1-1 / e) M^{*}\left(I_{0}\right) D_{n} \subseteq I_{1}
$$

Then

$$
(1-1 / e) M^{*}([0, u]) D_{n} \subseteq(1-1 / e)\left(\frac{R M^{*}([0, u])}{M_{0}}\right) D_{n} \subseteq \frac{I_{1}}{M_{0}} \subseteq \frac{K_{1}}{M_{0}}
$$

where the body $K_{1}$ is defined by:

$$
K_{1}:=M_{u_{N_{1}}} \ldots M_{u_{1}} K_{0}
$$

Since $M^{*}([0, u])=\frac{1}{2} \int_{S^{n-1}}\left|x_{1}\right| d \sigma(x) \approx \frac{1}{\sqrt{2 \pi n}}$, the proof is complete.

The inner radius $c_{2} / \sqrt{n}$ does not decrease under additional symmetrizations (it may increase). Next consider Minkowski symmetrizations which bring the convex hull of $K_{1}$ isometrically close to the ball. By Theorem 1.2 there exist $N_{2}=c n|\log (\varepsilon)|$ directions $u_{1}, \ldots, u_{N_{2}}$ such that

$$
(1-\varepsilon) D_{n} \subseteq M_{u_{N_{2}}} \ldots M_{u_{1}} \operatorname{conv}\left(\frac{K_{1}}{M_{0}}\right) \subseteq(1+\varepsilon) D_{n}
$$

We define the body $K_{2}$ to be:

$$
K_{2}:=M_{u_{N_{2}}} \ldots M_{u_{1}} K_{1}
$$

Note that Minkowski symmetrizations commute with the convex hull operation, i.e. $M_{u} \operatorname{conv} K=\operatorname{conv} M_{u} K$, simply because in general $\operatorname{conv}(A+B)=\operatorname{conv} A+\operatorname{conv} B$. Thus $\frac{K_{2}}{M_{0}}$ is a star shaped body whose convex hull is isometrically close to the ball. We use the following standard lemma to show that such a body must contain some $\delta$-net of the sphere. More precisely:

Lemma 2.2. Let $n \geq 2, \varepsilon \in(0,1)$, and let $K \in T^{n}$ be such that

$$
(1-\varepsilon) D_{n} \subseteq \operatorname{conv} K \subseteq(1+\varepsilon) D_{n}
$$

Then $K$ contains a $2 \sqrt{\varepsilon}$-net of the sphere $(1-\varepsilon) S^{n-1}$, that is

$$
(1-\varepsilon) S^{n-1} \subseteq K+2 \sqrt{\varepsilon} D_{n} .
$$


Proof. Let $x \in(1-\varepsilon) S^{n-1}$. We claim that the intersection $\left(x+2 \sqrt{\varepsilon} D_{n}\right) \cap K$ is not empty. Denoting the hyperplane supporting $(1-\varepsilon) D_{n}$ at the point $x$ by $H$ and the halfspace with boundary $H$ by $H^{+}=\{y \mid\langle x, x\rangle \leq\langle x, y\rangle\}$, we will show that $H^{+} \cap K \cap\left(x+2 \sqrt{\varepsilon} D_{n}\right)$ is not empty. Indeed,

$$
H^{+} \cap K \subseteq H^{+} \cap(1+\varepsilon) S^{n-1} \subseteq\left(x+2 \sqrt{\varepsilon} D_{n}\right),
$$

see Figure 1. The set $H^{+} \cap K$ is not empty (since $x \in\left(H^{+} \cap \operatorname{conv} K\right)$ ), and thus the proof is complete.

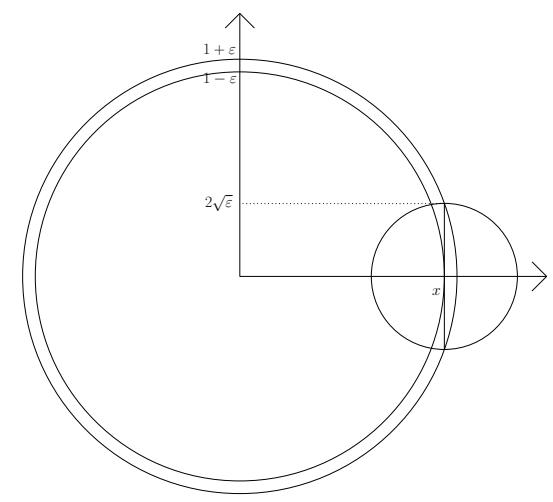

Figure 1: The small ball around $x$ contains all points of $K$ in $H^{+}$.

Note that in fact, since $K+2 \sqrt{\varepsilon} D_{n}$ is star shaped, we have:

$$
(1-\varepsilon) D_{n} \subseteq K+2 \sqrt{\varepsilon} D_{n} .
$$

The outer radius $1+\varepsilon$ does not increase under additional symmetrizations (it may decrease). As for the inner radius, we begin with the small ball obtained in Lemma 2.1. and use (3) to increase it geometrically. More precisely:

Lemma 2.3. Let $n \geq 2, K \in T^{n}, r \in(0,1)$, and $\varepsilon \in\left(0, \varepsilon_{0}\right)$, where $\varepsilon_{0}=1 / 25$. Assume that:

- $r D_{n} \subseteq K$.

- $(1-\varepsilon) D_{n} \subseteq K+2 \sqrt{\varepsilon} D_{n}$.

Then there exist $N=\alpha+\beta|\log \varepsilon|+\gamma|\log r|$ Minkowski symmetrizations transforming $K$ into $\tilde{K}$ satisfying

$$
(1-4 \sqrt{\varepsilon}) D_{n} \subseteq \tilde{K},
$$

where $\alpha, \beta, \gamma$ are positive constants. 
Proof. In each of the two cases $r<2 \sqrt{\varepsilon}$ and $2 \sqrt{\varepsilon} \leq r$, we argue a bit differently, so we handle them separately. We begin with the case of smaller initial inner radius.

Case a. Increasing $r$ geometrically to reach $2 \sqrt{\varepsilon}$ :

If $r<2 \sqrt{\varepsilon}$ we may take the second assumption and write for any $u \in S^{n-1}$ :

$$
r \frac{1-\varepsilon}{2 \sqrt{\varepsilon}} D_{n} \subseteq \frac{r}{2 \sqrt{\varepsilon}} K+r D_{n} \subset K+r D_{n} \subseteq K+R_{u}(K),
$$

So that $r \frac{1-\varepsilon}{4 \sqrt{\varepsilon}} D_{n} \subseteq M_{u}(K)$. Defining the (decreasing) function $q:(0,1) \rightarrow \mathbb{R}^{+}$by $q(\varepsilon)=\frac{1-\varepsilon}{4 \sqrt{\varepsilon}}$, we have $q(\varepsilon) \geq q\left(\varepsilon_{0}\right)=6 / 5$, so the inner radius multiplies by at least $6 / 5$. If $2 \sqrt{\varepsilon} \leq r(6 / 5)^{m}$, then after $m$ symmetrizations the inner radius reaches $2 \sqrt{\varepsilon}$. Thus after $N_{a}=4+3\left|\log \left(\frac{\varepsilon}{r^{2}}\right)\right|$ symmetrizations, we have reduced to the second case, where $2 \sqrt{\varepsilon} \leq r$.

\section{Case b. Increasing $r$ geometrically towards 1:}

Again, for any $u \in S^{n-1}$ we have

$$
\begin{aligned}
& \frac{(1-[2 \sqrt{\varepsilon}+\varepsilon])+r}{2} D_{n}=\frac{(1-\varepsilon) D_{n}+(r-2 \sqrt{\varepsilon}) D_{n}}{2} \subseteq \\
& \frac{K+2 \sqrt{\varepsilon} D_{n}+(r-2 \sqrt{\varepsilon}) D_{n}}{2}=\frac{K+r D_{n}}{2} \subseteq M_{u}(K) .
\end{aligned}
$$

So the difference $(1-[2 \sqrt{\varepsilon}+\varepsilon])-r$ decreases by at least half. The inner radius exceeds $1-4 \sqrt{\varepsilon}$ if and only if its difference from $(1-[2 \sqrt{\varepsilon}+\varepsilon])$ decreases below $2 \sqrt{\varepsilon}-\varepsilon>\sqrt{\varepsilon}$. Thus it suffices to decrease that difference to $\sqrt{\varepsilon}$. For that we require no more than $N_{b}=\left|\log _{2}(\sqrt{\varepsilon})\right|=\frac{1}{\log 4}|\log (\varepsilon)|$ symmetrizations.

Proof of Theorem 1.3. To complete the proof one has to combine the steps above. Let $K \in T^{n}$, such that $M^{*}(K)=1$. By Lemma 2.1, there exist cn Minkowski symmetrizations that transform $K$ into a set $K_{1}$ such that

$$
\frac{c_{2}}{\sqrt{n}} D_{n} \subset K_{1}
$$

and no Minkowski symmetrization can change this fact. Let $0<\varepsilon<1 / 25$. By Theorem 1.2, there exist $c n|\log \varepsilon| \operatorname{symmetrization}$ that transform $\operatorname{conv}\left(K_{1}\right)$ into a convex set $L$ such that $(1-\varepsilon) D_{n} \subset L \subset(1+\varepsilon) D_{n}$. Since $M_{u} \operatorname{conv} K=\operatorname{conv} M_{u} K$, we may apply the same symmetrizations to $K_{1}$ to obtain a new set $K_{2}$ such that $\operatorname{conv}\left(K_{2}\right)=L$. By Lemma 2.2, we get the following:

$$
(1-\varepsilon) S^{n-1} \subset K_{2}+2 \sqrt{\varepsilon} D_{n} .
$$

In addition,

$$
\frac{c_{2}}{\sqrt{n}} D_{n} \subset K_{2}
$$


Thus, by Lemma 2.3 there exist $\alpha+\beta|\log \varepsilon|+\gamma \log n$ Minkowski symmetrizations that transform $K_{2}$ into $K_{3}$ such that

$$
(1-4 \sqrt{\varepsilon}) D_{n} \subset K_{3}
$$

Recall that $K_{3} \subset(1+\varepsilon) D_{n}$. To sum it up, we applied no more than

$$
c n+c n|\log \varepsilon|+\alpha+\beta|\log \varepsilon|+\gamma \log n \leq C n|\log \varepsilon|
$$

symmetrizations, for some universal constant $C>0$.

During the proof we assumed that $\varepsilon<1 / 25$. This can be easily changed to $\varepsilon<1 / 2$, at the cost of a different constant in the expression $C n|\log \varepsilon|$, by always symmetrizing the set to be $\varepsilon / 25$ close to the Euclidean ball. By the same argument

one may extend for all $\varepsilon \in(0,1)$, and the corresponding bound on the number of symmetrizations will become $n\left(C|\log \varepsilon|+C^{\prime}\right)$.

\section{References}

[1] Bourgain J., Lindenstrauss J., Milman V.D., Minkowski Sums and Symmetrizations. Geometric Aspects of Functional Analysis, Lecture Notes in Mathematics, Vol. 1317, 44-66 (1988).

[2] Klartag B., Rate of Convergence of Geometric Symmetrization. Geom. and Funct. Anal. (GAFA), Vol. 14, Issue 6, 1322-1338 (2004).

Dan Itzhak Florentin, danflorentin@gmail.com

Department of Mathematics, Weizmann Institute of Science, Rehovot, Israel.

Alexander Segal, segalale@gmail.com

School of Mathematical Science, Tel Aviv University, Tel Aviv, Israel. 